\title{
A New Geometric Approach to Explain the Features of the Micro World
}

\author{
Pavel A. Stabnikov \\ Nikolaev Institute of Inorganic Chemistry SB RAS, Novosibirsk, Russia \\ Correspondence to: Pavel A. Stabnikov, stabnik@niic.nsc.ru
}

Keywords: Wave-Particle Duality, Geometry Postulates, Integral Transformations, Interference

Received: May 31, $2019 \quad$ Accepted: July 20, $2019 \quad$ Published: July 23, 2019

Copyright (c) 2019 by author(s) and Scientific Research Publishing Inc.

This work is licensed under the Creative Commons Attribution International License (CC BY 4.0).

http://creativecommons.org/licenses/by/4.0/

(c) (i) Open Access

\section{ABSTRACT}

A novel geometric approach is proposed for the development of the wave-particle notions. This approach is based on a comparison of the two geometries with different sizes of an infinitesimal point. It is assumed that the smaller is object mass, the larger is the size of the infinitesimal point in comparison with the point of the geometry of macro world. Within this approach, the smaller is object mass, the larger is the uncertainty of its position from the viewpoint of macro objects (macro geometry). This approach provides a natural explanation of Heisenberg's indeterminancy principle. Formally, this approach appears as an unusual operation with an infinitesimal value (point). However, it should be noted that unusual operations (though with infinitely large values) are already known in physics. These are unattainability of the absolute zero of temperature and unattainability of the maximal velocity of movement. Interconnection of the two geometries with different sizes of infinitesimal values is possible with the help of the direct and inverse Weierstrass transformation. At present, diffraction effects are described using the wave notions about the light and Fourier transform. The diffraction of light is usually registered at a distance not less than 1 3 metres between the screens in one of which there is a slit or several slits. This distance is about $10^{6}$ times longer than the wavelength of the radiation. In the present work, an approach is proposed that allows one to describe the light fluxes at short distances between the screens with the help of Fourier and Weierstrass transforms.

\section{INTRODUCTION}

According to Heisenberg's indeterminancy principle, the geometry of micro world does not differ from our usual macro geometry but the uncertainty of the simultaneous determination of such corpuscular characteristics as coordinate $(x)$ and pulse $(p)$, pulse time and energy etc. is introduced. Any pair of these characteristics is connected by relation $\Delta x \Delta p \geq h$, where $h$ is the Planck constant. This relation 
shows that the smaller is the error of the determination of one of the values ( $x$ or $p)$, the larger is the error for another one. This approach is generally accepted [1]. Two approaches were proposed almost a hundred years ago to describe the movement and properties of the objects with such features: matrix mechanics by Heisenberg, and wave mechanics by Schrödinger. Both of these approaches lead to the same results [2]. However, in Heisenberg's matrix mechanics the problem is in excluding all non-observable quantities (velocities and trajectories of particles, for example electrons) keeping only observables (discrete transitions in atoms). However, within this approach it is impossible to establish the physical sense of calculation itself, while solving Schrödinger's wave equation one obtains the wave function; the squared function is the probability for a particle to occur in a definite site of the space and time. In other words, the wave function allows visualizing atomic processes as the wave phenomena. The wave function and its square may be plotted, which makes it understandable for human perception. This is what defined the extensive development of Schrödinger's wave mechanics.

However, another interpretation is possible when the space of microparticles differs from usual geometry by the increased value of infinitesimal (point). The description of the movement of micro objects is usual, but indistinctness arises as a result of the transfer of information about movement into the geometry with a smaller infinitesimal value (to the macro level). With this approach, the difference between the objects in two geometries is reduced to different sharpness of figures or images. This is how image blurring arises during the transformation from micro geometry into macro. This allows us to explain Heisenberg's indeterminancy principle in a natural way. The most important feature is that this geometric approach is more fundamental (it is based on comprehensible geometric statements) than the wave-particle duality. However, this approach assumes a paradoxical change of the idea of infinitesimal. In other words, it is proposed to assign the attribute of infinitesimal geometrically to a finite quantity (illegible point). This approach allows us to describe the interference of light at close distances from the screen.

\section{UNATTAINABILITY OF THE INFINITE AND FINITE}

Only infinitely large or infinitely remote quantities possessed unattainability in classical physics, because it is impossible to reach infinitely large values. In early representations, body temperature could have any values from $-\infty$ to $+\infty$. However, Lord Kelvin proposed to transfer the minimal temperature to a finite value $\left(-273.15^{\circ} \mathrm{C}\right)$ and accept this value to be equal to zero. In this case, many thermodynamic expressions are written in a simpler form. In fact, this approach is the transfer of an infinitely remote value $(-\infty$ for temperature) to the zero of Kelvin's scale. This approach also automatically transfers unattainability of the infinitely far point into the zero of Kelvin's scale, and this value becomes unattainable. An additional effect of this transfer is a decrease in the heat capacity of bodies almost to zero while temperature approaches the zero of Kelvin's scale. Otherwise unattainability of Kelvin's zero would be impossible. According to this approach, a body may be cooled to a temperature approaching the zero of Kelvin's scale but this final (finite) value cannot be achieved. This is the manifestation of unattainability attribute.

Another finite unattainable value for anybody with non-zero mass is the velocity of light. Ancient philosophers (with rare exception) thought that the velocity of light is infinite. The fact that the velocity of light is a finite value was established for the first time by $\mathrm{O}$. Roemer in 1676 on the basis of the observations of a satellite of Jupiter. The Earth rotating around the Sun may approach Jupiter or move away from it. A consequence is the difference in the times of shadowing of Jupiter's satellite. This effect allowed one to determine the velocity of light for the first time. Further on, the velocity of light was determined more precisely many times with the help of special devices allowing more accurate measurements of the distance and time.

In 1905, Einstein developed the Special Theory of Relativity (SR) to adjust the laws of classical mechanics and electrodynamics. According to this theory, the velocity of light measured in any inertial reference system is the same and independent of the motion of the system and the irradiator. According to SR, the velocity of light is the maximal velocity with unattainability attribute. The consequences of unattainability for the maximal velocity are the following statements: relativistic law of velocity composition, time 
dilation, and a decrease in the linear size of a moving body with respect to the chosen inertial system. Otherwise unattainability of the finite velocity of light would be impossible. According to SR, the velocity of any body may be very close to the velocity of light in vacuum but this limit cannot be exceeded.

\section{UNATTAINABILITY INFINITELY SMALL}

These two examples illustrate the simplicity of nature description with the help of so unusual approximations of infinitely large values to finite values. From the viewpoint of philosophy, not only infinitely large values but also infinitesimals possess unattainability. For instance, mathematical zero is reciprocal of infinitely large value. The attribute of the unattainability of the infinitely small was suggested in the aporias of the ancient Greek philosopher Zeno (for example, about Achilles and the tortoise). So, the idea of rendering unattainability to a small value is quite reasonable. It was demonstrated in $[3,4]$ that this approach allows theoretical substantiation of quantum effects during the transformation of data between the two geometries differing from each other by the values of infinitesimals.

The mathematical interconnection between the displays of two geometries with different infinitesimals may be built with the help of the direct and inverse integral Weierstrass' transformations [5]:

$$
F(t)=\frac{1}{\sqrt{4 \pi}} \int_{-\infty}^{+\infty} \mathrm{e}^{\frac{-(x-t)^{2}}{4}} f(x) \mathrm{d} x \quad f(x)=\frac{1}{i \sqrt{4 \pi}} \int_{s-i \infty}^{s+i \infty} \mathrm{e}^{\frac{(t-x)^{2}}{4}} F(t) \mathrm{d} t
$$

At present, computer numerical modeling with the help of Weierstrass transformation may be carried out with the help of computers. Solutions of some problems of light diffraction with the help of the integral Weierstrass transformation were described in $[3,4]$.

\section{DESCRIPTION OF LIGHT DIFFRACTION BY INTEGRAL TRANSFORMS}

Diffraction was explained in literature [6-9]. Let us use Figure 40 from [6] and Figure 6.2 from [7]. We see that the diffraction patterns are registered at a distance of $2-3 \mathrm{~m}$ from the first screen with a slit. Diffraction result is easily explained with the help of the wave representation of light. However, nothing is written about diffraction at a distance shorter than $1 \mathrm{~m}$. We will try to calculate diffraction effects (light fluxes) for the distances within this range. If the distance between the screens is short, the general picture of the light flux should depict the shapes and dimensions of slits (corpuscular approach). For longer distance between the screens ( $1 \mathrm{~m}$ and longer), diffraction effects should be manifested (the wave approach). To simulate the corpuscular approach, we will use Weierstrass transformation with the index in the core of the integral transformation $\frac{-(x-t)^{2} * L}{4 * \sigma}$, where $\mathrm{L}$ is the distance between the screens $(\mathrm{m}), \sigma$ is the relative size of an infinitesimal point. It was accepted in calculations that $\frac{x}{\sigma}=4 \mathrm{~m}^{-1}$.

The application of Weierstrass transformation to the model with two slits is shown in Figure 1 (plot a). This is a corpuscular contribution into the light flux. It follows from Figure 1 that total intensity gets smoothed out and scattered as the distance from the first screen increases ( $m$-metres). To describe diffraction, we will use Fourier transform (Figure 2).

The joint picture of the light flux on the registering screen was determined as a linear sum of corpuscular and wave scattering taking into account the total energy of incoming light. It was found that the corpuscular contribution decreases with an increase in the distance between the screens, while the wave contribution increases (Figures 3-5).

It follows from Figures 3-5 that using the integral Fourier and Weierstrass transformations it is possible to describe the light flux at a shorter distance between the screens than the distance described in textbooks. This approach is based on the idea that the motion of micro objects may be described by another geometry differing from the classical one by an increased size of the infinitely small point. 


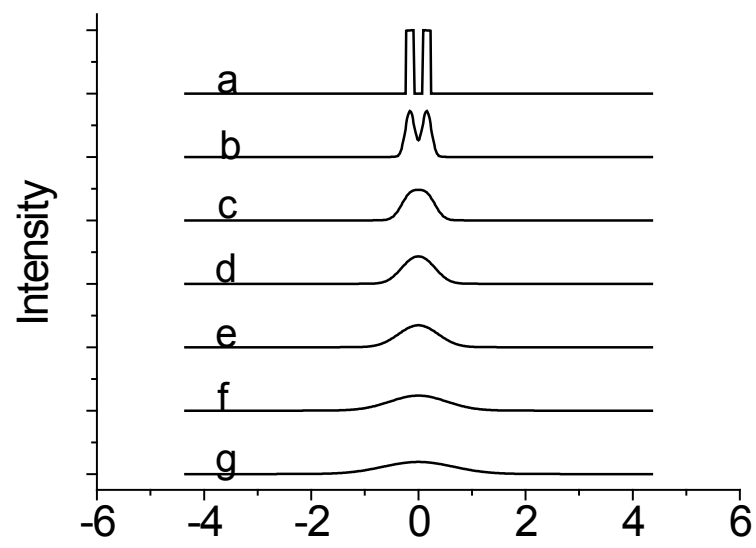

Figure 1. $a-0 \mathrm{~m} ; \mathrm{b}-0.01 \mathrm{~m} ; \mathrm{c}-0.05 \mathrm{~m} ; \mathrm{d}-0.1 \mathrm{~m}$; $\mathrm{e}-0.2 \mathrm{~m} ; \mathrm{f}-0.5 \mathrm{~m} ; \mathrm{g}-0.8 \mathrm{~m}$.

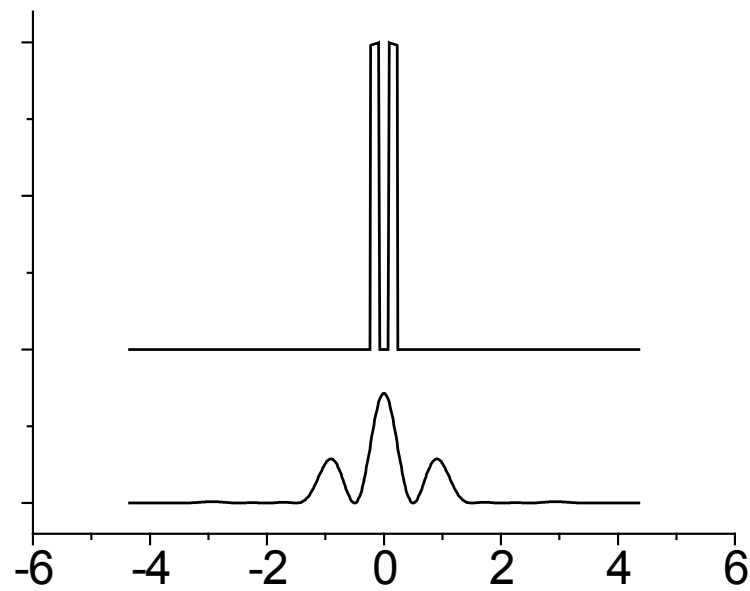

Figure 2. A symmetrical original with two slits and normalized square cosine of Fourier transform. This is the wave contribution into the light flux.

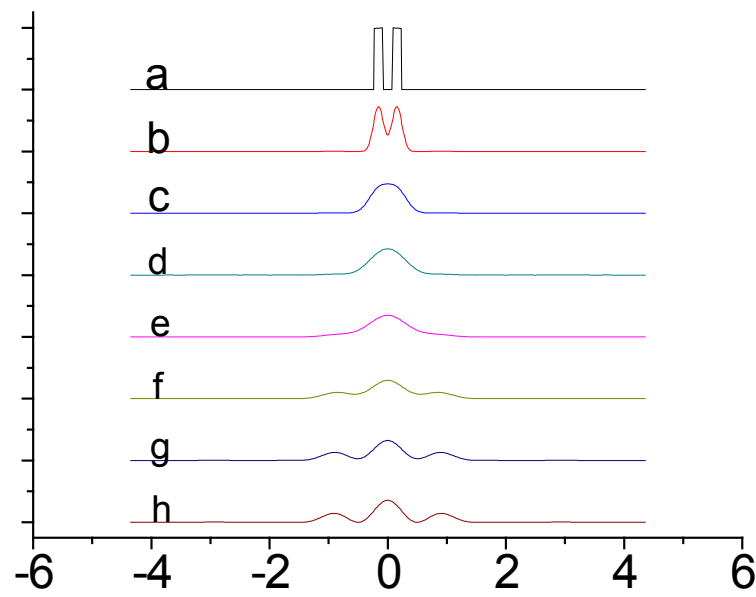

Figure 3. $a-0 \mathrm{~m} ; \mathrm{b}-0.01 \mathrm{~m} ; \mathrm{c}-0.05 \mathrm{~m} ; \mathrm{d}-0.1 \mathrm{~m}$; $\mathrm{e}-0.2 \mathrm{~m} ; \mathrm{f}-0.5 \mathrm{~m} ; \mathrm{g}-0.8 \mathrm{~m} ; \mathrm{h}-1 \mathrm{~m}$. 


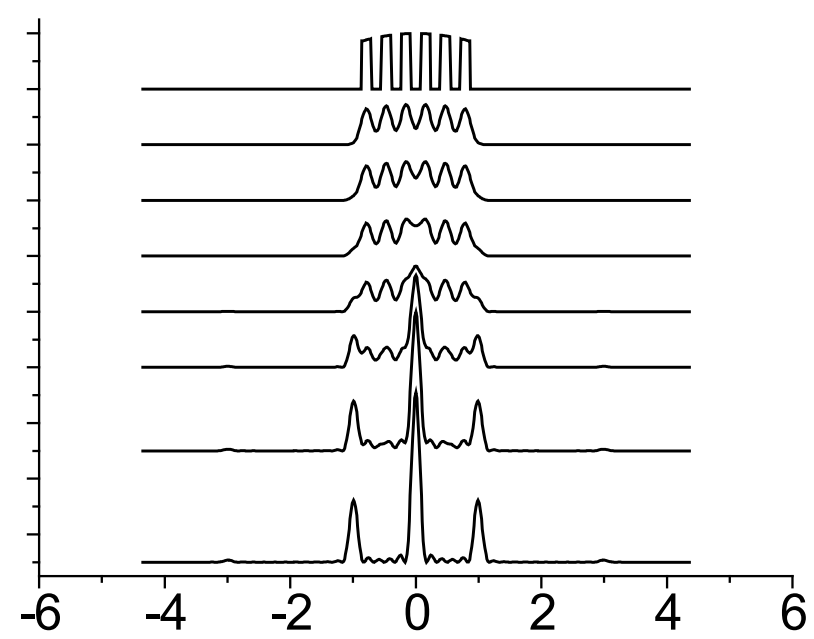

Figure 4. $\mathrm{a}-0 \mathrm{~m} ; \mathrm{b}-0.01 \mathrm{~m} ; \mathrm{c}-0.05 \mathrm{~m} ; \mathrm{d}-0.1$ $\mathrm{m} ; \mathrm{e}-0.2 \mathrm{~m} ; \mathrm{f}-0.5 \mathrm{~m} ; \mathrm{g}-0.8 \mathrm{~m} ; \mathrm{h}-1 \mathrm{~m}$.

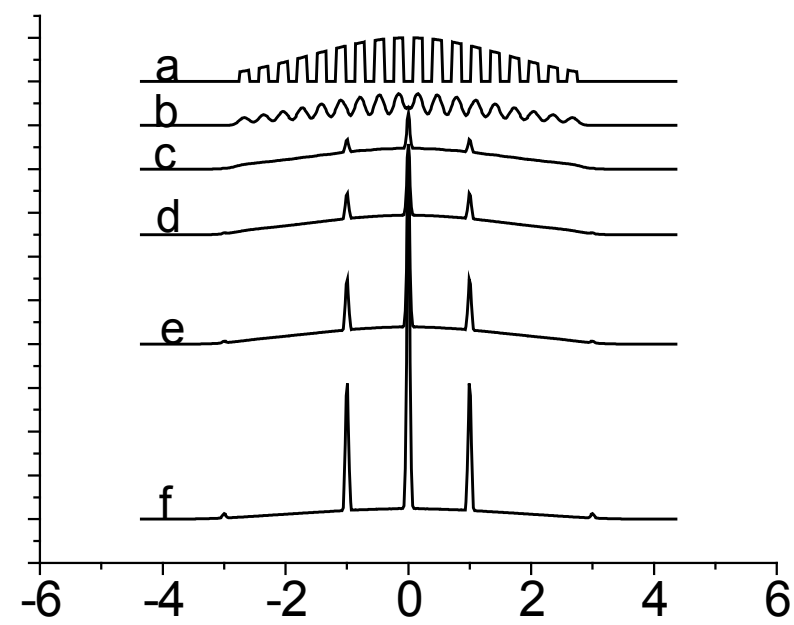

Figure 5. a-0 m; b-0.01 m; c-0.05 m; d-0.1 $\mathrm{m} ; \mathrm{e}-0.2 \mathrm{~m} ; \mathrm{f}-0.5 \mathrm{~m}$.

\section{CONCLUSION}

A new approach is proposed in the present work to explain the specific features of the microworld. This approach is based on the ideas of different metrics for infinitesimal in two geometries. This approach allows a simple explanation of Heisenberg uncertainty principle. An interconnection between two geometries with different infinitely small values may be established through the integral Weierstrass transform. It is demonstrated that the description of light interference requires both the corpuscular (Weierstrass transform) and wave (Fourier transform) description of light fluxes. This approach allows us to describe light fluxes at any distance from a screen with slits. This agrees with the approach proposed by D. Bohm [10], who separated the wave function into the corpuscular and wave components. Very paradoxically, but this also allowed him to describe light fluxes at any distance from the screen with slits.

\section{CONFLICTS OF INTEREST}

The author declares no conflicts of interest regarding the publication of this paper. 


\section{REFERENCES}

1. Feynman, R., Leighton, R. and Sands, M. (1963) Feynman Lectures of Physics. Vol. 3.

2. De Broglie, L. (1965) La Physique Nouvelle et les Quanta. ATOMIZDAT, Moscow, 231 p. (In Russian)

3. Stabnikov, P.A. (2018) Frames in Which Matter Develops. Palmarium Academic Publishing, Saarbrücken. (In Russian)

4. Stabnikov, P.A. and Babailov, S.P. (2019) Types of Interactions and Material Islands of Stability: From the Micro World to the Universal Scale. IIC SB RAN, Novosibirsk, 6,5 p. (In Russian)

5. Brychkov, Yu.A. and Prudnikov, A.P. (1977) Integral'nye preobrazovaniya obobshchennykh funktsiy. Nauka, Moscow, 288 p. (In Russian)

6. Pohl, R.W. (1963) Optik und atomphysik. Springer Verlag, Berlin, 525 p.

7. Ditchburn, R.W. (1965) Light. Glasgow, London, 631 p. (In Russian)

8. Landsberg, G.S. (1976) Optics. Moscow, 759 p. (In Russian)

9. Novotny, L. and Hecht, B. (2006) Principles of Nano-Optics. Cambridge University Press, Cambridge, 482 p.

10. Bohm, D. (1952) A Suggested Interpretation of the Quantum Theory in Terms of Hidden Variables. Parts I and II. Physical Review, 85, 166-193. 